\title{
FRIEDRICH A. VON HAYEK E IL PROBLEMA DELLA SOLIDARIETÀ
}

\author{
DARIO ANTISERI*
}

Resumen. La competizione nello sviluppo della scienza e nella vita di una democrazia costituisce la piü alta forma di collaborazione, cosí come lo é nell'economia di mercato - sistema económico che sta a base delle liberta politiche e che é la fonte maggiormente sicura del piü esteso benessere. La Grande Societá, tuttavia, é seriamente minacciata - ammonisce Hayek dalla riaffermazione dell»'etica tribale» del socialismo: «il concertó di 'giustizia sociale' é stato il cavallo di Troia tramite il quale ha fatto il suo ingresso il totalitarismo». Con ció Hayek non nega affatto il valore della solidarietá, in quanto la Grande Societá puó permettersi di aiutare i piü deboli e deve farlo.

Abstract. In the development of science and of a democracy, competition represents the highest form of collaboration. The same applies in the free market economic system that supports political freedom and corresponds to the most secure source of extended welfare. However, Hayek warns that The "Great Society" is seriously threatened by the comeback of the socialism's «tribal ethic»: "the concept of 'social justice' has been the Trojan horse for the entrance of the totalitarism». By saying this, he does not deny the value of solidarity. The Great Society can allow itself to help those in need, and actually it must do it.

* Libera Università degli Studi Sociali (LUISS), Roma. 


\section{SCIENZA, DEMOCRAZIA E MERCATO: UNITI DAL GRANDE PRINCIPIO DELLA «COMPETIZIONE»}

La ricerca scientifica può venir correttamente vista, nella scia del razionalismo critico, come un processo senza fine di soluzioni di problemi attraverso la «concorrenza» di più idee; sul piano politico, la ricerca scientifica trova il suo equivalente nella democrazia in quanto concorrenza tra le varie proposte dei differenti partiti; in economia la competizione si ha tra prodotti esposti sul mercato. Ricerca scientifica, democrazia e mercato funzionano in base al grande principio della concorrenza. Se nella vita intellettuale non c'è concorrenza tra idee, l'esito sarà un intollerante e cieco dogmatismo; se nella vita politica non c'è concorrenza, l'esito scontato sarà il totalitarismo; se in economia viene abolita la concorrenza del mercato, avremo una pianificazione centralizzata, fonte di oppressione, di corruzione e di dissipazione delle risorse.

La realtà è che, analogamente a quanto accade nella scienza, anche sul mercato vince - di volta in volta, e non certo per l'eternità - chi ha saputo inventare e produrre una «merce» che risolve un problema (magari prima inesistente) meglio di altre merci. E come nella scienza sovrani sono i fatti, così sul mercato (non truccato, senza protezioni) sovrani sono i consumatori con le loro preferenze, preferenze illuminate da valori scelti. Il mercato è un processo di soluzione di problemi. Ma esso è anche, o soprattutto, «un processo di esplorazione in cui gli individui cercano nuove opportunità che, una volta scoperte, possono essere usate anche da altri» (Hayek, 1988b, p. 320; e si veda anche Hayek, 1986, pp. 441-444). Chi abbraccia l'economia di mercato è antistatalista e antinterventista, vede nella proprietà privata dei mezzi di produzione il fondamento del benessere e della più ampia libertà, e considera la concorrenza come la più alta forma di collaborazione. Competizione, infatti, deriva dal verbo cum-petere, che significa cercare insieme: cercare insieme in 
maniera concorrenziale, come nella scienza così nel mercato, la migliore soluzione («teoria»o «merce»). La logica di mercato è una logica sensibile, ultrasensibile, ai problemi degli utenti o consumatori. Già in questo primo senso è una logica della solidarietà.

\section{IL «PROCESSO» AL MERCATO SI BASA SU DI UNA «ISTRUTTORIA»SBAGLIATA}

Ma è proprio il valore della solidarietà quello a cui ci si richiama a destra e a sinistra e a cui fanno appello tanti amici cattolici per scagliarsi contro l'economia di mercato. L'economia di mercato —si dice- è esattamente 1'opposto della solidarietà. La competizione viene vista come una guerra che, ovviamente, emargina i vinti. Il profitto sarebbe semplicemente un furto. Il mercato si aggiunge- è spietato: schiaccia tutto e tutti e non si accorge nemmeno dell'esistenza di persone che, come per esempio i portatori di handicap, nella competizione non possono neppure pensare di entrare. E le critiche diventano un rifiuto secco e totale del mercato allorché si punta il dito su quell'orribile cosa che è la compra-vendita di armi micidiali. Il «mercato» delle armi è uno degli argomenti più usati contro l'economia di mercato.

Non è proprio il caso di porre in discussione le buone intenzioni di quanti - cattolici o no- sono contrari al mercato in nome della solidarietà con i più umili, con gli svantaggiati, con quanti hanno urgente bisogno di cure indispensabili e di aiuto. Ma, come ben si sa, di buone intenzioni son lastricate le vie dell'inferno. C'è un vecchio detto, in Pennsylvania, secondo il quale i Quaccheri andarono nel Nuovo Mondo per fare del bene e finirono per fare soldi. Scriveva Thoreau nel 1845: «Non c'è odore peggiore di quello della bontà andata a male [...] Se sapessi per certo che qualcuno sta venendo a casa mia col deliberato consenso di farmi del bene, scapperei a gambe levate». Ed ecco come 
Milton Friedman (Friedman, 1975, p. 82) commenta questo pensiero di Thoreau: «chiunque è libero di fare del bene, ma a spese sue».

Non è, dunque, questione di intenzioni. Queste le guarderà Iddio. Per noi contano, dalla prospettiva politica, gli esiti di tali progetti intenzionali. E i risultati del rifiuto del mercato in nome della solidarietà sono e sono stati letteralmente disastrosi, sia per la libertà che per il benessere di milioni e milioni di uomini. Senza economia di mercato, senza la proprietà privata dei mezzi di produzione, non è possibile nessuna vera democrazia, nessuno Stato di diritto, e nessuna libertà individuale è garantita. Lo Stato che ha abolito la proprietà privata dei mezzi di produzione è lo Stato che possiede tutti i mezzi; e «chi possiede tutti i mezzi stabilisce tutti i fini» (Hayek, 1948, p. 81; e Hayek, 1996, p. 163). E, d'altro canto, «la libertà di stampa è un puro inganno se l'autorità statale controlla tutti gli uffici-stampa, tutte le tipografie e tutte le cartiere» (Mises, 1959, p. 277).

L'economia di mercato è la base della libertà politica. Ma essa è anche la fonte più sicura del benessere più esteso, in quanto è proprio l'economia di mercato che si è rivelata come lo strumento più adeguato, tra quelli disponibili, per produrre ricchezza per il maggior numero di persone (Hayek, 1997b, pp. 196-218; e Hayek, 1967, p. 23 e ss.). Di conseguenza, se non vogliamo che la solidarietà significhi un reciproco pianto sulle nostre miserie (o, peggio ancora, lo storno - cioè il furto- di risorse da chi produce a clientes parassiti i quali per mestiere fanno gli «elettori»), allora dobbiamo asserire - perché intellettualmente convinti e moralmente motivati- che è l'economia di mercato a configurarsi come un vero e proprio strumento della solidarietà. Certo, una società che abbia abbracciato l'economia di mercato non è e non sarà mai il paradiso. E, tuttavia, è decisamente preferibile dividere in parti diseguali la ricchezza in un mondo di libertà e di pace piuttosto che dividere in parti sempre e comunque diseguali la miseria in un mondo di oppressione in 
cui necessariamente vige il principio per cui «chi non ubbidisce non mangia».

Si guarda al mercato delle armi e si rifiuta la logica del mercato. E' forse questo un valido argomento? Sarebbe come dire che occorre abolire la scienza perché la fisica ha scoperto l'energia nucleare e la chimica ci ha fatto conoscere gli effetti del curaro. Ma è chiaro che se uno usa il curaro per uccidere un'altra persona, la colpa non è né del curaro né della scienza; colpevole è soltanto l'assassino e malvagia è la sua etica. Analogamente, se uno realizza profitto vendendo armi, il colpevole non è il «mercato»; colpevoli sono coloro che vendono e comprano armi e disumana è la loro etica. Il mercato è sempre innocente.

\section{F.A. VON HAYEK: LA GRANDE SOCIETÀ È MINACCIATA DALL'«ETICA TRIBALE»}

Tutta la nostra conoscenza è ipotetica, congettuale; resta smentibile. Questo è il grande insegnamento di Karl R. Popper. Un insegnamento decisamente contro-intuitivo: giacché ognuno di noi pensa di avere l'esatta percezione della situazione, la vera soluzione dei problemi, i valori più alti razionalmente fondati, l'ideologia «scientifica», il «vero» Dio o l'indiscutibile dimostrazione della non-esistenza di Dio. Ebbene, se l'insegnamento di Popper è contro-intuitivo, il pensiero di Hayek è controistintivo: giacché egli sostiene che la Grande società è minacciata dalla riaffermazione dell'»etica tribale» del socialismo. Una delle ragioni per cui in tempi recenti si è potuto notare un forte ritorno del modo di pensare dell'organizzazione e un declino della comprensione del funzionamento dell'ordine di mercato sta nel fatto - dice Hayek - che «una parte in continuo aumento dei membri della società lavora come membro di vaste organizzazioni e trova il proprio orizzonte di comprensione limitato 
a ciò che è richiesto dalla struttura interna di tali organizzazioni medesime» (Hayek, 1986, p. 344). In realtà, «una parte sempre crescente passa l'intera vita lavorativa come membro di vaste organizzazioni ed è portata a pensare soltanto in termini delle esigenze del modo di vita organizzativo» (ibid.).

Le cose, però, non si fermano qui: quando gruppi particolari pensano che i loro membri meritano questo o quest'altro ed hanno la forza di imporre queste loro idee -impedendo agli altri di offrire i loro servizi a condizioni più favorevoli-, si avrà allora che «ogni gruppo di azioni del genere dirette ad assicurare un reddito o una posizione particolare di propri membri crea un ostacolo all'integrazione della Grande società ed è quindi antisociale nel vero senso della parola. Diventa una forza disgregante perché non produce una riconciliazione ma un conflitto fra gli interessi dei diversi gruppi. Come ben sanno i partecipanti attivi alla lotta per la 'giustizia sociale', questa diventa in pratica una lotta per il potere da parte di interessi organizzati in cui le argomentazioni di giustizia servono unicamente come pretesti» (Hayek, 1986, p. 347). L'esperienza mostra ad abundantiam che «le azioni collettive di gruppi organizzati sono quasi invariabilmente in contrasto con l'interesse generale». E qui Hayek va alla radice della sua argomentazione: «Sono i sentimenti ereditati dalle forme più antiche di società che portano a condannare come antisociale il perseguimento degli interessi individuali che contribuiscono all'interesse generale, e ad elogiare come 'sociale' l'asservimento a quegli interessi settoriali che distruggono l'ordine globale» (Hayek, 1986, p. 349; Hayek, 1997b, pp. 33-39). Gli interessi organizzati di gruppi sociali forti danneggeranno i membri di gruppi meno forti. 


\section{UNA PROPOSTA «CONTRO-ISTINTIVA»}

Nel piccolo gruppo - all'interno del quale gli uomini sono vissuti per centinaia di migliaia di anni- domina uno scopo comune tangibile. Gli individui del piccolo gruppo si conoscono, si aiutano; tra loro c'è solidarietà, lealtà personale. Ben diversamente stanno le cose nella Grande società: "Il sorgere dell'ideale della giustizia impersonale basata su norme formali è stato raggiunto attraverso una continua lotta contro i sentimenti di lealtà personale che sono alla base della società tribale, ma che nella Grande Società non devono essere lasciati influenzare l'uso dei poteri coercitivi del governo» (Hayek, 1986, p. 354). E ciò per la ragione che «l'estensione» graduale di un comune ordine pacifico dal piccolo ordine alla comunità sempre più vasta ha comportato scontri continui tra le richieste di una giustizia settoriale basata su scopi comuni tangibili e i requisiti della giustizia universale, egualmente applicabili all'estraneo come al membro del gruppo» (ibid.). Da qui - precisa Hayek«un costante conflitto tra le emozioni profondamente radicate nella natura umana attraverso millenni di esistenza tribale, e le richieste di principi astratti di cui nessuno afferra appieno il significato. Le emozioni umane sono legate ad oggetti concreti, e le sensazioni di giustizia, in particolare, sono ancora strettamente connesse ai bisogni tangibili del gruppo a cui ognuno appartiene, alle necessità del mestiere o della professione, del clan o del villaggio, della città o del paese al quale ognuno appartiene. Soltanto una ricostruzione mentale dell'ordine globale della Grande società ci permette di capire che il tendere consapevolmente verso propositi concreti e comuni, che a molti ancora appare più meritevole e superiore rispetto alla cieca osservanza di norme astratte, distruggerebbe quel sistema più vasto in cui tutti gli esseri umani contano allo stesso modo» (ibid). 
Nell'ordine di mercato «i produttori sono spinti a servire la gente senza conoscere i suoi bisogni individuali». Ma lavorare per soddisfare i bisogni di altri uomini sconosciuti «presuppone e richiede concetti morali diversi da quelli secondo i quali la gente serve fini tangibili. Farsi guidare indirettamente dall'attesa di un vantaggio monetario, il quale opera come indicatore delle richieste da parte di terzi, richiede nuove concezioni morali che non prescrivono fini particolari, ma piuttosto norme che limitino lo spettro delle azioni permesse. Divenne parte dell'ethos della società aperta il fatto che fosse meglio investire il proprio patrimonio in strumenti che rendessero possibile produrre di più a costi inferiori piuttosto che distribuirlo tra i poveri, o prendersi cura dei bisogni di migliaia di persone sconosciute piuttosto che provvedere ai bisogni di pochi vicini conosciuti. Ovviamente, queste idee non si svilupparono perché coloro che le seguirono per primi capivano che in tal modo conferivano maggiori benefici ai loro simili, ma perché i gruppi e le società che così agivano prosperavano più delle altre; di conseguenza, ciò si trasformò gradualmente nel 'dovere' morale riconosciuto della 'vocazione' a farlo» (Hayek, 1986, pp. 355-356).

Si pensa, di solito, che fare del bene implichi obbligatoriamente di occuparsi di specifici bisogni di persone conosciute; ad esempio, «che sia molto meglio aiutare un uomo conosciuto che muore di fame piuttosto che alleviare i bisogni acuti di un centinaio di persone sconosciute» (Hayek, 1986, p. 356). Hayek la pensa diversamente: a parte il problema di che cosa intenda fare un imprenditore di successo con i propri profitti - dopo averli guadagnati- questo imprenditore, precisa con forza Hayek - «è portato a beneficiare più persone mirando al maggior utile possibile di quanto potrebbe fare se si concentrasse sul soddisfacimento dei bisogni di persone conosciute. Egli è condotto dalla mano invisibile del mercato a portare il soccorso delle comodità moderne alle più povere case che non conosce nemmeno» (ibid). 
Scopi moralmente ispirati possono produrre conseguenze immorali. Il concetto di «giustizia sociale» —insiste Hayek— «è stato il cavallo di Troia tramite il quale ha fatto il suo ingresso il totalitarismo» (Hayek, 1986, p. 346). E, in effetti, «bisogna rendersi conto [...], che gli ideali del socialismo (o della giustizia sociale) che a tal riguardo appaiono così allettanti, non offrono veramente una nuova morale ma fanno unicamente appello a istinti ereditati da un tipo di società più antico. Essi rappresentano un atavismo, un tentativo vano di imporre alla società aperta la morale della società tribale; se tale morale prevalesse, non soltanto distruggerebbe la Grande società ma minaccerebbe la sopravvivenza di quei grandi numeri che l'umanità ha raggiunto grazie a tre secoli di ordine di mercato» (Hayek, 1986, p. 338). E ancora: «Il socialismo è semplicemente la riaffermazione di quell'etica tribale il cui graduale indebolimento aveva reso possibile la grande società» (Hayek, 1986, p. 341).

\section{LA FUNZIONE DELLE ASSOCIAZIONI VOLONTARIE}

Da siffatte considerazioni Hayek non inferisce che le norme di condotta che fanno riferimento a rapporti personali speciali «abbiano perso importanza nel funzionamento della Grande società». E qui egli porta il suo discorso sull'enorme importanza delle associazioni volontarie. " $\mathrm{E}^{\prime}$ totalmente estranea ai principi base di una società libera l'idea secondo la quale tutto ciò di cui il pubblico ha bisogno debba essere soddisfatto da organizzazioni obbligatorie, e tutti i mezzi che gli individui sono disposti a devolvere ai fini pubblici debbano essere sotto controllo del governo. Al contrario, il vero liberale deve auspicare il maggior numero possibile di quelle società particolari all'interno dello stato, quelle organizzazioni volontarie che si 
pongono tra l'individuo e lo stato, che il falso individualismo di Rousseau e la Rivoluzione francese vollero sopprimere. Egli vuole però privarle di qualsiasi potere esclusivo e obbligatorio. Il liberalismo non è individualistico nel senso di 'ognuno per sé', sebbene sia per natura sospettoso verso la tendenza delle organizzazioni ad arrogarsi diritti esclusivi per i propri membri» (Hayek 1986, p. 362).

Con queste sue riflessioni Hayek intende sottolineare «l'importanza dell'esistenza di numerose associazioni volontarie non soltanto per gli scopi particolari di coloro che condividono un interesse comune ma anche per fini pubblici nel vero senso della parola. Lo stato dovrebbe avere il monopolio della coercizione necessaria a limitare la coercizione stessa; ciò non significa che lo stato debba avere l'esclusivo diritto di perseguire fini pubblici. In una società veramente libera, gli affari pubblici non si limitano a quelli di governo (men che meno a quelli del governo centrale), e lo spirito pubblico non dovrebbe esaurirsi nell'interesse verso lo stato» (ibid). E sempre Hayek insiste: «Una delle grandi debolezze della nostra epoca è la mancanza di fede e pazienza per costruire organizzazioni volontarie per gli scopi che si hanno a cuore. Si chiede immediatamente al governo di far uso della coercizione (o con i mezzi raccolti tramite coercizione) per avere qualsiasi cosa appaia desiderabile a molti. Tuttavia nulla può avere un effetto più negativo sulla partecipazione reale del cittadino di quanto lo sia il fatto che lo stato, invece di fornire soltanto il quadro di riferimento essenziale per una crescita spontanea, diventa monolitico e si prende cura di tutti i bisogni che possono essere soddisfatti soltanto dallo sforzo concentrato di molti. Il grande merito dell'ordine spontaneo, che si occupa soltanto dei mezzi, è l'aver reso possibile l'esistenza di numerose comunità volontarie a servizio della scienza, delle arti, dello sport e così via. E' altamente auspicabile che nel mondo moderno questi gruppi tendano ad estendersi oltre i confini nazionali, e che ad esempio uno scalatore svizzero possa avere più cose in 
comune con uno scalatore giapponese che con un tifoso di calcio del suo paese, e che possa appartenere ad un'associazione comune con il primo, completamente indipendente da qualsiasi organizzazione politica a cui entrambi appartengono» (Hayek, 1986, p. 363). Da ciò ben si comprende, allora, che «l'attuale tendenza dei governi a portare tutti gli interessi comuni di vasti gruppi sotto il proprio controllo tende a distruggere il vero spirito pubblico. Come risultato, un numero sempre crescente di uomini e donne si sta allontanando dalla vita pubblica, a cui in passato avrebbe dedicato molte energie. Sul continente europeo l'eccessivo interessamento dei governi ha in passato ostacolato lo sviluppo di organizzazioni volontarie per fini pubblici, e ha prodotto una tradizione in cui gli sforzi privati erano spesso considerati come ingerenze gratuite. Gli sviluppi moderni sembrano aver prodotto progressivamente una situazione simile anche nei paesi anglosassoni, dove un tempo gli sforzi privati per fini pubblici erano una caratteristica tipica della vita sociale» (ibid.).

\section{HAYEK E LA DIFESA DEI PIÙ DEBOLI}

Il capitalismo non è parola di Dio. Gli Stati o le società che hanno abbracciato l'economia di mercato non sono il paradiso. Eppure, dove vige l'economia di mercato si è avuta l'espansione più ampia delle libertà politiche: Stato di diritto ed economia di mercato nascono, vivono e muoiono insieme. Dove vige l'economia di mercato regna il più ampio benessere: negli Stati capitalistici viene «divisa» in modo diseguale la ricchezza.

$\mathrm{Ma}$ - e qui sorge la domanda più urgente- che ne è della solidarietà all'interno di uno Stato che abbia abbracciato l'economia di mercato? In Legge, legislazione e libertà Hayek scrive: «(...) Il capitalismo come esiste oggi ha indubbiamente molti 
difetti rimediabili, che un'intelligente politica di libertà dovrebbe correggere. Un sistema che si basa sulle forze spontanee del mercato, dopo aver raggiunto un certo livello di ricchezza, non è indubbiamente incompatibile con uno stato che fornisca, fuori dal mercato, qualche forma di previdenza in caso di estrema povertà» (Hayek, 1986, p. 346). La Grande società - precisa Hayek - «è riuscita brillantemente ad abolire la povertà in senso assoluto. Nei paesi sviluppati nessuno in grado di lavorare è privo oggi di cibo e casa; mentre per chi è incapace di guadagnare abbastanza da se stesso queste cose essenziali sono fornite generalmente fuori dal meccanismo di mercato. La povertà in senso relativo deve naturalmente continuare ad esistere in una società che non sia completamente egualitaria: fintanto che esiste l'ineguaglianza qualcuno deve essere in fondo alla scala. Ma l'abolizione della povertà assoluta non è aiutata dallo sforzo di raggiungere la «giustizia sociale». Di fatto, in molti paesi in cui la povertà assoluta è ancora un problema grave, la preoccupazione della 'giustiza sociale' è diventata uno dei maggiori ostacoli all'eliminazione della povertà. In Occidente, il raggiungimento da parte delle masse di un benessere ragionevole è stato causato dall'aumento generale di ricchezza ed è stato solo rallentato dalle misure che interferiscono con il meccanismo di mercato. E' stato proprio quest'ultimo a creare l'aumento del reddito globale, che a sua volta ha reso possibile provvedere fuori dal mercato al sostentamento di chi non era in grado di guadagnare abbastanza. I tentativi di 'correggere' i risultati del mercato verso la 'giustizia sociale' hanno probabilmente prodotto più ingiustizia sotto forma di nuovi privilegi, ostacoli alla mobilità e frustrazione di sforzi, di quanto abbiano contribuito ad alleviare la sorte dei poveri» (Hayek, 1986, pp. 349-350).

Hayek è persuaso che il servizio postale statale è del tutto inefficiente; propone l'abolizione del monopolio monetario statale («che è stato usato per defraudare e ingannare i cittadini» [Hayek, 1986, p. 431; e Hayek, 1978]); combatte il monopolio 
statale delle trasmissioni televisive e della scuola. E, tuttavia, egli, sempre in Legge, legislazione e libertà, scrive: «Lungi dal propugnare 'uno stato minimo', riteniamo indispensabile che in una società avanzata il governo debba usare il proprio potere di raccogliere fondi per le imposte per offrire una serie di servizi che per varie ragioni non possono essere forniti -o non possono esserlo in modo adeguato-dal mercato» (Hayek, 1986, p. 416). In tal modo, afferma Hayek, molte delle comodità capaci di rendere tollerabile la vita in una città moderna vengono fornite dal settore pubblico: «la maggior parte delle strade [...], la fissazione degli indici di misura, e molti altri tipi di informazione che vanno dai registri catastali, mappe e statistiche, ai controlli di qualità di alcuni beni e servizi» ( Hayek, 1986, 418).

Per Hayek, la sfera delle attività di governo non vincolate da leggi di governo è molto vasta. Certo, l'esigere il rispetto della legge, la difesa dai nemici esterni, il campo delle relazioni estere sono attività dello Stato. Ma c'è sicuramente di più, poiché "pochi metteranno in dubbio che soltanto questa organizzazione [dotata di poteri coercitivi: lo Stato] può occuparsi delle calamità naturali quali uragani, alluvioni, terremoti, epidemie e così via, e realizzare misure atte a prevenire o rimediare ad essi» (Hayek, 1986, p. 428). Ed è ovvio, allora, "che il governo controlli dei mezzi materiali e sia sostanzialmente libero di usarli a propria discrezione» (ibid).

Ma vi è - e qui le considerazioni che seguono sono di estrema importanza e smentiscono parecchie interpretazioni affrettate e certamente non documentate del pensiero di Hayek-, «vi è ancora - scrive Hayek - tutta un'altra classe di rischi rispetto ai quali è stata riconosciuta solo recentemente la necessità di azioni governative, dovuta al fatto che, come risultato della dissoluzione dei legami della comunità locale e degli sviluppi di una società aperta e mobile, un numero crescente di persone non è più strettamente legato a gruppi particolari su cui contare in caso di disgrazia. Si tratta del problema di chi, per varie ragioni, 
non può guadagnarsi da vivere in un'economia di mercato, quali malati, vecchi, handicappati fisici e mentali, vedove e orfani cioè coloro che soffrono condizioni avverse, le quali possono colpire chiunque e contro cui molti non sono in grado di premunirsi da soli, ma che una società la quale abbia raggiunto un certo livello di benessere può permettersi di aiutare» (ibid; cfr Hayek, 1948, p. 107).

Ha ben ragione Angelo M. Petroni allora a scrivere che: «Non ci si stancherà mai di ripetere come Hayek sia sempre stato in favore della creazione di una 'rete' di sicurezza che permettesse di vivere a coloro che non fossero stati capaci di provvedere al proprio sostentamento all'interno del mercato. Ma ciò deve avvenire fuori dal mercato non come 'correzione' del mercato medesimo» (Petroni, 1992, p. 18).

Una società che abbia abbracciato la «logica di mercato» può permettersi il conseguimento di fini umanitari perché è ricca; e può farlo tramite operazioni fuori mercato e non con manovre che siano correzioni del mercato medesimo. Ma ecco quella che, ad avviso di Hayek, è la ragione per cui essa deve farlo: "Assicurare un reddito minimo a tutti, o un livello sotto cui nessuno scenda quando non può più provvedere a se stesso, non soltanto è una protezione assolutamente legittima contro rischi comuni a tutti, ma è un compito necessario della Grande Società in cui l'individuo non può rivalersi sui membri del piccolo gruppo specifico in cui era nato» (Hayek, 1986, p. 429; e Hayek, 1988b, p. 102). E, in realtà, ribadisce Hayek, «un sistema che invoglia a lasciare la relativa sicurezza goduta appartenendo ad un gruppo ristretto, probabilmente produrrà forti scontenti e reazioni violente, quando coloro che ne hanno goduto prima i benefici si trovino, senza propria colpa, privi di aiuti, perché non hanno più la capacità di guadagnarsi da vivere» (Hayek, 1986, p. 429; e Hayek, 1988a, p. 102)). 


\section{LEONE XIII E LE RAGIONI \\ DELLA DIFESA DELLA «PROPRIETÀ PRIVATA»}

Leone XIII, in un periodo storico in cui la proprietà privata dei mezzi di produzione veniva considerata come un furto sia dalla stragrande maggioranza degli intellettuali che dalle masse organizzate nei partiti di ispirazione marxista e socialista, fece della proprietà privata una difesa lucida e coraggiosa; e con grande preveggenza additò i pericoli distruttivistici insiti nell'abolizione della proprietà privata. Leggiamo nella Rerum Novarum: «La comunanza dei beni proposta dal socialismo va assolutamente respinta, perché nuoce a quegli stessi che si vogliono aiutare, offende i diritti naturali di ciascuno, altera le funzioni dello Stato e turba la pace comune».

Va a grande merito di Leone XIII aver affermato che talvolta le buone intenzioni possono avere conseguenze diametralmente opposte a quelle intese: i socialisti intendono abolire la proprietà privata al fine di raggiungere il maggior benessere per il maggior numero di persone; l'esito di questa loro azione non potrà essere che l'immiserimento progressivo di tutti e la più crudele schiavitù per i popoli. Questo prevedeva Leone XIII nella Rerum Novarum del 1891. Questo prevederà Ludwig von Mises negli anni Venti. E questo è quanto si è tragicamente verificato nel secolo passato. E, dunque, è etico il socialismo»? E' davvero solidale ed etico il socialismo»? Basta, sul serio, l'etica dell'intenzione?

No! L'etica dell'intenzione è certamente necessaria; ma essa non è politicamente e socialmente sufficiente. Lo statalismo e l'interventismo, possiamo ammettere che, almeno nei più, siano state e siano concezioni animate dalle più serie intenzioni di solidarismo, da attenzione alle difficoltà di sfortunati ed infelici. I risultati, però, cui hanno portato le politiche stataliste ed interventiste e le pratiche paternalistiche sono disastrosi. Ecco, allora, che l'etica della responsabilità — l'etica che cioè guarda ai risultati-si impone sull'etica dell'intenzione. 
È proprio perché hanno abbracciato l'etica della responsabilità che, ai nostri giorni, cattolici come Michael Novak e padre Robert Sirico negli Stati Uniti d'America, Jean-Yves Naudet e Jacques Garello in Francia, don Angelo Tosato in Italia, si sono persuasi che è esattamente l'economia di mercato a costituire lo strumento più adeguato per il conseguimento della solidarietà. $\mathrm{E}$, prima di costoro è necessario fare menzione di cattolici liberali come A. de Tocqueville, F. Bastiat, A. Rosmini, W. Röpke, K. Adenauer, L. Einaudi e L. Sturzo.

VIII. SUL FRONTE DI IERI:

\section{PENSATORI CATTOLICI SOSTENITORI} DELLA «LOGICA DI MERCATO»

Alexis de Tocqueville (1805-1859): «Quello che sopra ad ogni altro caratterizza ai miei occhi i socialisti di tutti i colori, di tutte le scuole, è una sfiducia profonda per la libertà, per la ragione umana, un profondo disprezzo per l'individuo preso in se stesso, al suo stato di uomo; ciò che li caratterizza tutti è un tentativo continuo, vario, incessante, per mutilare, per raccorciare, per molestare in tutti i modi la libertà umana; è l'idea che lo Stato non debba soltanto essere il direttore della società, ma debba essere, per così dire, il padrone di ogni uomo; il suo padrone, il suo precettore, il suo pedagogo [...]; in una parola, è la confisca, in un grado più o meno grande, della libertà umana».

Frédéric Bastiat (1801-1850): «Quando una nazione è oppressa da tasse, niente è più difficile -io direi pure impossibile-che ripartirle in maniera equa». E poi: «Allorché si sarà ammesso in via di principio che lo Stato ha l'incarico di operare in modo fraterno in favore dei cittadini, si vedranno tutti i cittadini trasformarsi in postulanti. Proprietà fondiaria, agricoltura, industria, commercio, marina, compagnie industriali, tutti si agiteranno per reclamare i favori dello Stato. Il tesoro pubblico 
sarà letteralmente saccheggiato. Ciascuno troverà buone ragioni per dimostrare che la fraternità legale deve essere intesa in questo senso: 'I vantaggi per me ed i costi per gli altri [...]'. Lo sforzo di tutti tenderà a strappare alla legislazione un lembo di privilegio fraterno».

Antonio Rosmini (1797-1855). Leggiamo nella Filosofia del diritto: «La proprietà esprime veramente quella stretta unione di una cosa con una persona (...). Questa specie di unione che si chiama proprietà cade sempre dunque tra la persona e la cosa e racchiude un dominio di quella sopra di questa. La proprietà è il principio della derivazione dei diritti e dei doveri giuridici. La proprietà costituisce una sfera intorno alla persona, di cui la persona è il centro; nella qual sfera niun altro può entrare». Da ciò l'imperativo di rispettare l'altrui proprietà. Per Rosmini, asserisce Clemente Riva, «la persona (...) ha sempre ragione di fine e non può mai essere quantificata nella sua altissima dignità». E ancora: «Le persone sono principio e fine dello Stato. Sono esse che costituiscono, che assegnano lo scopo e i limiti, per cui lo Stato e tutti gli organi statali sono dei semplici mezzi per le persone che ne sono realmente il fine. Di qui il carattere ministeriale o di servizio dell'autorità (...). Per questo il bene pubblico, come bene dell'organismo statale deve essere subordinato al bene privato di ciascuna persona. E il bene comune non è altro che il bene privato e proprio di tutte le singole persone, o quel bene generale a cui ciascuna realmente partecipa».

Wilhelm Röpke (1898-1916): "Antichità classica e Cristianesimo, entrambi sono i veri antenati del liberalismo, perché sono gli antenati di una filosofia sociale che regola il rapporto, ricco di contrasti, tra l'individuo e lo Stato secondo i postulati di una ragione inserita in ogni uomo e della dignità che spetta ad ogni uomo come fine e non come mezzo, e così contrappone alla potenza dello Stato i diritti di libertà del singolo». «Il liberalismo non è [...] nella sua essenza un abbandono del 
Cristianesimo, bensì è il suo legittimo figlio spirituale [...] Il liberale diffida di ogni accumulazione di potere, perché sa che di ogni potere, che non viene tenuto nei suoi limiti da contrappesi, si fa presto o tardi abuso».

Konrad Adenauer (1876-1967): «La media proprietà è una sicurezza essenziale degli stati democratici». «Una socializzazione troppo vasta rende troppo grande l'accumulazione della potenza nelle mani dello Stato e avevamo personalmente sofferto i pericoli che ne derivano per la vita di un popolo. Il socialismo porta necessariamente alla sottomissione dei diritti e della dignità dell'individuo allo Stato o a una collettività simile allo Stato». "Il nazional-socialismo non era altro che la conseguenza spinta fino al crimine [...] del ludibrio del valore dell'individuo, risultante dall'ideologia materialista». «Il concetto della supremazia, della onnipotenza dello Stato, del suo primato sulla libertà e sulla dignità dell'individuo è in contraddizione con la legge della natura dei cristiani. Secondo il mio parere, l'esistenza e il rango dell'individuo devono venire prima dello Stato».

Luigi Einaudi (1874-1961). In una delle sue Prediche inutili Luigi Einaudi domandava: «I vescovi hanno adempiuto bene all'ufficio di curare nei seminari l'educazione economica dei giovani che sentono la vocazione del sacerdozio? Hanno procurato che si impartissero ai seminaristi le nozioni elementari necessarie per distinguere tra il lecito giuridico e il dovere caritativo, fra l'economia e la morale?».

Luigi Sturzo (1871-1959). Scriveva don Luigi Sturzo il 4 novembre del 1951: «Oggi si è arrivati all'assurdo di voler eliminare il rischio per attenuare le responsabilità fino ad annullarle [...] Gli amministratori, i direttori, gli esecutori degli enti statali sanno in partenza che se occorrono prestiti, garantisce lo Stato; se occorre lavoro dovrà trovarlo lo Stato; se si avranno perdite si ricorrerà allo Stato; se si produce male ripara lo Stato; se non si conclude un gran che, i prezzi li mantiene alti lo Stato. 
Dov'è il rischio? svaporato. E la responsabilità? svanita. E l'economia? compromessa [...] In Italia, oggi, solo le aziende dei poveri diavoli possono fallire: le altre sono degne di salvataggio, entrando per questa porta a far parte degli enti statali, parastatali e pseudo-statali. Il rischio è coperto in partenza, anche per le aziende che non sono statali, ma che hanno avuto gli appoggi dello Stato. In un Paese, dove la classe politica va divenendo impiegatizia [...]; dove la classe economica si statalizza; dove la classe salariale va divenendo classe statale, non solo va a morire la libertà economica, ma pericola la libertà politica [...]». Il 13 maggio del 1954 Sturzo asseriva: «La Pira crede che il problema da risolvere sarebbe quello di arrivare alla totalità del sistema finanziario in mano allo Stato [...] Questo io chiamo statalismo, e contro questo dogma io voglio levare la mia voce senza stancarmi finché il Signore mi darà fiato; perché sono convinto che in questo fatto si annidi l'errore di far dello Stato l'idolo: Moloch o Leviathan che sia [...]». E il 27 maggio 1954: «[...] La mia difesa della libera iniziativa è basata sulla convinzione scientifica che l'economia di Stato non solo è anti-economica ma comprime la libertà e per giunta riesce meno utile, o più dannosa, secondo $\mathrm{i}$ casi, al benessere sociale».

\section{PENSATORI CATTOLICI LIBERALI DEI NOSTRI GIORNI}

Robert Sirico, sacerdote cattolico americano e membro della Mont Pélerin Society, ha fondato nell'aprile del 1990 il Lord Acton Institute con l'intento che "dimostrando l'indissolubile nesso tra religione e libertà, leaders economici di fede religiosa possano forgiare un'alleanza in grado di garantire e sostenere una società libera e onesta». Scrive padre Sirico: «Prima di poter essere consumata, la ricchezza dev'essere prodotta; la povertà è la condizione naturale della razza umana, il risultato dell'ostacolo 
frapposto dalla natura al benessere umano: la scarsità. L'abbondanza è l'eccezione: non c'è bisogno di far nulla per 'produrre' povertà, laddove tanto bisogna fare per produrre ricchezza (...) Pertanto, prima di poter soccorrere i poveri, dobbiamo indagare sulla natura della produzione di ricchezza». E poi: «Il cristianesimo trova la sua realtà più vera quando vive nel cuore di persone che, in virtù di un impegno liberamente assunto, contribuiscono a modificare in meglio il mondo circostante. I momenti più oscuri del cristianesimo si sono avuti quanto la Chiesa ha voluto governare dai parlamenti e dalle capitali degli uomini politici, anziché nel cuore dei fedeli». «Se, come avvenuto negli ultimi vent'anni, la Chiesa alla ricerca della verità ha potuto dialogare coi pensatori influenzati da Marx, allora forse è maturo il tempo perché si apra il dialogo con il liberalismo classico».

Jean Yves Naudet (nato nel 1948) è professore di scienze economiche presso la Facoltà di diritto e scienze politiche di AixMarseille III. I regimi del socialismo reale — fa presente Naudet- si sono caratterizzati prima di tutto per la loro inefficienza. «Ma tale inefficienza - egli scrive- proveniva dal rifiuto di riconoscere la natura dell'uomo e i suoi diritti, a cominciare da quello di perseguire il proprio fine personale, e ancora dal diritto di proprietà o dal diritto di iniziativa economica». Il mercato, tuttavia, «deve rimanere al suo posto». L'economicismo utilitarista è semplicemente disumano e «la Centesimus annus, in conformità con l'intera dottrina sociale della Chiesa, fa ben vedere che il mercato non è che uno strumento, e non un fine». E «la dottrina sociale della Chiesa, così come si configura da un secolo a questa parte e come viene proposto ancora oggi nella Centesimus annus, implica una chiara priorità in favore della famiglia».

Jacques Garello (nato nel 1934), professore di Storia delle idee e dei fatti economici all'Università di Aix-en-Provence, allievo di Daniel Villey e Jacques Rueff, è tra i rappresentanti più prestigiosi della scuola liberale francese. Garello è persuaso della 
sicura convergenza tra liberalismo e pensiero cattolico. L'ostilità della Chiesa nei confronti dell'economia di mercato è dipesa, ad avviso di Garello, dal fatto che il liberalismo di ieri si presentava con credenziali inaccettabili per la Chiesa cattolica. Tali caratteristiche del liberalismo erano la fede smisurata nella Ragione, l'utilitarismo e il materialismo dell'homo oeconomicus. Ma, oggi - grazie al lavoro scientifico della Scuola austriaca di economia e soprattutto grazie al pensiero di F.A. von Hayeksi è sviluppato un pensiero liberale che ha rotto nella maniera più netta con la tradizione razionalista-utilitarista-materialista. Con questo sono cadute, appunto, le ragioni dell'ostilità della Chiesa nei confronti del liberalismo. La verità, afferma Garello, è che le virtù morali che accompagnano il mercato «sono quelle dell'Occidente cristiano»: 1'onestà, il rispetto della parola data, il rispetto degli altri, il rifiuto dell'oppressione e dell'arbitrio. «Coniugando liberalismo e cattolicesimo, l'Occidente ritrova e ritroverà il suo equilibrio intellettuale, morale e spirituale».

Angelo Tosato (nato nel 1938 e prematuramente scomparso, nel 1999), sacerdote dal 1963, ha insegnato presso l'Università Gregoriana e presso la Lateranense, esegeta di fama internazionale, ha introdotto in Italia il pensiero di M. Novak ed è autore del volume: Economia di mercato e cristianesimo (1994). Qui di seguito soltanto due sue considerazioni: «Anche in campo cattolico si sente l'esigenza di riesaminare la tradizionale condanna del capitalismo, di sottoporre a verifica la presunta incompatibilità tra i valori del sistema di economia politica liberale e l'etica cristiana. Il processo di ripensamento può dirsi ormai avviato». «(...) Se per 'capitalismo' si intende (...) onesta ricerca del benessere personale, familiare e collettivo, e più ancora un sistema che dia a tutti la facoltà di esercitare liberamente e proficuamente la propria capacità in campo economico, allora il rapporto col cristianesimo non può che essere di sintonia e di collaborazione».

Michael Novak (nato nel 1933 in Pennsylvania) è il teologoeconomista americano, autore de Lo spirito del capitalismo 
democratico e il cristianesimo (1982). Ecco, di seguito, alcune sue considerazioni: «La democrazia politica (...) è compatibile solo con un economia di mercato»; «Il capitalismo non sarà in grado di far sparire completamente il cancro della povertà né a livello internazionale né a livello locale, ma, grazie ad esso, la povertà sarà sicuramente meno diffusa di quanto non sia attualmente nei paesi socialisti o nel Terzo Mondo. L'unione di capitalismo e democrazia non porterà il regno dei Cieli sulla Terra ma, per liberare i poveri dalla miseria e dalla tirannia e per dar spazio alla loro creatività, il capitalismo e la democrazia possono fare molto di più di quanto sia in potere di tutte le altre alternative esistenti»; "Nell'intimo cuore del sistema capitalista vi è la fiducia nelle capacità creative dell'uomo. Come affermano i teologi cattolici, e come provato dall'esperienza, questa fiducia è ben riposta (...) Dal Vecchio e dal Nuovo Testamento abbiamo imparato che, per provvedere ai nostri bisogni e ai nostri desideri, dobbiamo agire personalmente senza aspettare l'aiuto dello Stato (...) In questo modo abbiamo imparato a vivere come uomini e donne liberi, responsabili del nostro destino. E se pure ci inginocchiamo innanzi a Dio, di fronte al mondo stiamo a testa alta e ci facciamo carico delle nostre responsabilità personali». ${ }^{1}$

${ }^{1}$ I brani di Leone XIII, A. de Tocqueville, F. Bastiat, A. Rosmini, W. Roepke, K. Adenauer, L. Einaudi, L. Sturzo, R. Sirico, J.-Y. Naudet, J. Garello, A. Tosato, M. Novak vengono citati dai testi richiamati in bibliografia - testi tutti rinvenibili nell'antologia: Cattolici a difesa del mercato, a cura di Dario Antiseri, SEI, Torino, 1995. 


\section{BIBLIOGRAFIA}

HAYEK, F.A., 1967, Storia e politica, in AA.VV., Il capitalismo e gli storici, trad. it., Sansoni, Firenze.

- 1969, La società libera, trad. it., Vallecchi, Firenze; (nuova edizione, SEAM, Roma, 1998).

- 1948, Verso la schiavitù, trad. it., Rizzoli, Milano, (nuova edizione, con il titolo: La via della schiavitù, Rusconi, Milano, 1995).

- 1986, Legge, legislazione e libertà, trad. it., Il Saggiatore, Milano.

- 1998, Studi di filosofia, politica ed economia, Rubbettino, Soveria Mannelli.

- 1997a, L'abuso della ragione, trad. it., SEAM, Roma; (precedente edizione italiana, con lo stesso titolo, presso Vallecchi, Firenze, 1967).

- $\quad$ 1997b, La presunzione fatale, trad.it., Rusconi, Milano.

- 1978, Denationalization of Money. The Argument Refined, Institute of Economic Affairs, $2^{a}$ ed., London.

- 1988a, Nuovi studi di filosofia, politica, economia, storia delle idee, trad.it., Armando, Roma.

- 1988b, La concorrenza come procedura per la scoperta del nuovo, in Conoscenza, mercato e pianificazione, a cura di E. Donzelli, Il Mulino, Bologna.

- 1996, Hayek su Hayek, a cura di S. Kresge e L. Wenar, trad. it., Ponte alle Grazie, Firenze.

FrIEDMAN, M., 1975, Contestazione liberale, trad. it., Sansoni, Firenze.

Cubeddu, R., 1995, Friedrich A. von Hayek, Borla, Roma.

PETRONI, A.M., 1992, L'impossibilità di redistribuire la libertà degli uomini, in «L'Opinione», giugno 1992.

Mises, L., 1959, L'azione umana, trad. it., UTET, Torino, 1959.

LEONE XIII, 1891, Rerum novarum.

Tocqueville, A. de, 1848, Discorso sul diritto al lavoro, in Scritti politici, 1, trad. it., a cura di N. Matteucci, UTET, Torino, 1969. 
BASTIAT, F., 1848, Giustizia e fraternità, in F. Bastiat e G. Molinari, Contro lo statalismo, trad. it., a cura di C. Lottieri, Liberilibri, Macerata, 1994.

Rosmini, A., 1865, Filosofia del diritto, 1, Tipografia di Paolo Bertolotti, Intra.

RÖPKE, W., 1951, Crisi e rinnovamento del liberalismo, in La crisi del collettivismo, trad. it., di C. Antoni e M. Biscione, La Nuova Italia, Firenze.

Adenauer, K., 1966, Memorie 1945-1953, trad. it., Mondadori, Milano.

EINAUDI, L., 1969, Prediche inutili, Einaudi, Torino.

STURZO, L., 1951-1954, Opera Omnia, Zanichelli, Bologna, vol. XII (4 novembre 1951); vol. XIII (13 maggio 1954).

NovaK, M., 1987, Lo spirito del capitalismo democratico e il cristianesimo, ed. it., a cura di A. Tosato, Studium, Roma.

SIRICO, R., 1991, Teologi alla ricerca della liberazione, in «Biblioteca della libertà», XXVI, pp. 19-32.

NAUDET, J.-Y., 1992, La liberté pour quoi faire? Centesimus annus et l'économie, trad. it., D. Antiseri in AA.VV., Cattolici a difesa del mercato, SEI, Torino, 1995, pp. 303-314.

Garello, J., 1994, Cattolicesimo e liberalismo, trad. it. di D. Antiseri, in AA.VV., Cattolici a difesa del mercato, cit., pp. 319-331.

TosATo, A., 1987, Cristianesimo e capitalismo (il problema esegetico di alcuni paesi evangelici), in Rivista biblica italiana, 35, 1987, pp. 465-476; e 1994, Economia di mercato e cristianesimo, Borla, Roma. 